\title{
Benefits and Risks of Using Blockchain in Smart Energy: A Literature Review
}

\author{
Emre Erturk \\ Eastern Institute of Technology, New Zealand \\ E-Mail: eerturk@eit.ac.nz \\ Dobrila Lopez \\ Eastern Institute of Technology, New Zealand \\ E-Mail: dlopez@eit.ac.nz \\ Wei Yang Yu \\ Eastern Institute of Technology, New Zealand \\ E-Mail: YUW3@student.eit.ac.nz
}

\begin{abstract}
Being a decentralized system makes Blockchain a suitable technology solution in many cases. The Smart Energy domain has the potential to benefit from the use of Blockchain, by improving data and transaction management, through applications such as peer-to-peer energy trading, grid enhancement, and electric vehicle support. Many researchers have proposed models and prototypes of blockchain-based applications, which are expected to improve the performance of smart energy systems. In this report, a qualitative literature review was performed to look into a sample of these application proposals and to try to identify the impacts of blockchain technology in Smart Energy. This resulted in the discovery of both positive and negative factors. The beneficial factors include increased security, improved data privacy, data transparency and immutability, removal of third-party control and trust, ubiquitous solution, and greater data accessibility. The list of challenges includes limited scalability and speed, off-chain support requirement, high establishment and maintenance cost, and requiring further practical tests. One of the interesting findings is that the impact of Blockchain technology as to whether it may increase or decrease transaction costs is still unclear. Finally, the paper recommends that a future study may focus on economic feasibility and transaction costs, and ascertain which Smart Energy cases and application designs
\end{abstract}


are more appropriate for Blockchain technology.

Keywords: Smart Energy, Energy Management, Blockchain Systems

\section{INTRODUCTION}

The potential of Blockchain technology has been noticed by varies industries. It is reasonable to hypothesize that the energy industry, especially smart energy, can also take advantage of introducing blockchain-based systems, to self-regulate and manage the contractual information and transactions.

The objective of this research is to investigate the impact of blockchain technology in smart energy systems from both positive and critical perspectives. To achieve this, existing proposals of blockchain-based smart energy systems will be analyzed to identify both benefits and potential risks and challenges presented in those approaches. The overall research question is - What are the impacts of the application of blockchain technology in smart energy?

The rest of this report is structured as follows. The Literature Review section provides the background on blockchain and smart energy, as well as existing smart energy applications that utilize blockchain technology. The Methodology section discusses the approaches and criteria used in this research. The Findings section describes the results of the analysis, identifying the major benefits and challenges. This analysis focuses on a target sample of 16 articles. These findings are further examined in the Discussion section. The Conclusion section summarizes the report and provides future research directions.

\section{LITERATURE REVIEW}

\section{Blockchain}

In short, blockchain is a decentralized system consisting of records of all transactions that ever happened among the participants, in other words, a public ledger (Swan, 2015; Yli-Huumo, Ko, Choi, Park, \& Smolander, 2016). A typical blockchain transaction works in the following way. The transaction is represented as a "block" with information stored within. The block is broadcast to every node in the network and waits for approval from the nodes that this transaction is valid. After the approval, the block is added to the existing blockchain and the transaction is completed (Crosby, Nachiappan, Pattanayak, Verma, \& Kalyanaraman, 2016).

According to Shen and Pena-Mora (2018), the three "pillars" of blockchain platforms are smart contracts, distributed consensus, and crypto token. This represents the business logic, database, and infrastructure of the platform respectively. The use of blockchain has evolved over the last decade, from blockchain 1.0 to 3.0 (Swan, 2015). 
Different 'versions' of blockchain focus on different industries, and they are all being used in different areas.

In Blockchain 1.0, the main application of blockchain is cryptocurrency. Bitcoin is one of the most popular cryptocurrencies in the industry. Bitcoin introduced two key components of blockchain: distributed consensus and crypto token. The distributed consensus mechanism introduced by Bitcoin is called proof-of-work (PoW), which is used to determine which node will be authorized to manage the chain (official validator). PoW requires nodes to invest a certain amount of computational power to complete a cryptographical calculation, and the first node to complete the question will be identified by the whole network and thus become the official validator (Nakamoto, 2008). In this system, there is also a reward for the efforts of the validators of new transactions (Shen \& Pena-Mora, 2018).

With Blockchain 2.0, this technology has started to be recognized by other business areas, and the idea of the Smart Contract was also introduced. One of the well-known products at this stage is Ethereum (Ethereum Foundation, 2019). The main difference between 2.0 and 1.0 is the introduction of the concept of the Smart Contract and a new distributed consensus. Unlike Bitcoin (which has a very limited level of programmability), Ethereum provides fully programmable smart contracts that can be utilized in various businesses (Swan, 2015). Ethereum has evolved its distributed consensus from a PoW to something called a proof-of-stake (PoS). In PoS, an official validator is selected based on the proportion of stakes held by the node. In practice, this has significantly reduced the computational power required, as a result of no longer requiring the solution of an algorithm (Shen \& Pena-Mora, 2018).

At the Blockchain 3.0 stage, this technology is used typically in areas other than finance. One example is Hyperledger Fabric (Linux Foundation, 2019). It features a permissioned blockchain system where only permissioned nodes can be added to the network and the identities of the nodes in the system are known. This is more suitable for general industrial and organizational uses. In a permissioned blockchain, a trusted identity management system is involved in managing the participants of the network. A decentralized database provides extra transparency and auditability, in comparison to a traditional database (Shen \& Pena-Mora, 2018). Based on the focus and role of the platform, permissioned blockchains may provide a different level of smart contract capabilities. Due to the open nature of permissionless blockchains, distributed consensus mechanisms in those blockchains may enhance system security. In a permissioned blockchain, identities of the nodes are known, and there is more freedom in picking a distributed consensus. Overall, a permissioned blockchain does not constantly require a crypto token, since the participation of nodes is supposed to be monitored. However, an application token can still be used for special purposes, 
depending on the application environment (Shen \& Pena-Mora, 2018).

\section{Smart Energy}

The idea of being "smart" has been drawing attention from both government and industry in recent years. This concept "encapsulates ideals of efficiency, security and utilitarian control in a technologically mediated and enabled environment" (Strengers, 2013, p.1). The "smart" tag has been added to different industries, including the smart energy sector. One of the most critical components of an energy system is the energy grid. Using advanced information communication technologies in multiple segments of the energy grid results in a Smart Grid (SG). The European Commission defined SG as "an electricity network that can intelligently integrate the actions of all users connected to it generators, consumers and those that do both in order to efficiently deliver sustainable, economical and secure electricity supplies" (Jenkins, Long, \& Wu, 2015). In comparison with the traditional energy grids designed mainly for fossil fuels, SG has a greater interest in renewable energy sources like wind and solar energy to help environmental efforts (Moretti et al., 2017).

\section{Blockchain-based Smart City Application}

In the report published by Xie et al. (2019), blockchain applications in the smart grid sector were categorized into four major types: electronic energy trading, enhancement of stability and data security in smart grid, renewable energy finance, and thing-to-thing electricity trading. Similarly, in another report by Shen and Pena-Mora (2018), three major sectors were mentioned about the application of blockchain in the smart energy area: grid security and meter transparency, peer-to-peer energy trading, and electric vehicles to grid systems. Based on these reports and the results from the literature, this report categorized the application of blockchain in the smart energy system into three types: peer-to-peer (P2P) energy trading, grid enhancement, and electric vehicle support.

\section{P2P Energy Trading}

The increasing amount of rooftop solar panels deployed in many areas has been shifting the role of many energy users from consumers to "prosumers", who act as both producers and consumers (El Rahi, Saad, Glass, Mandayam, \& Poor, 2016). Data stored on the blockchain is public and always available while remaining pseudonymous, which provides both transaction transparency and user anonymity. Besides, the decentralized nature of blockchain makes it highly resistant to denial of service attacks. Furthermore, the immutability of the data on the blockchain makes it a trusted technology for a P2P energy trading platform (Kounelis et al., 2017).

Aitzhan and Svetinovic (2018) proposed a decentralized P2P energy trading 
system involving blockchain technology. Their system is token-based, featuring anonymous price negotiation between peers, and secure trading transactions. The researchers performed security analysis and performance evaluation through a set of case studies. While the overall performance of the design appeared satisfying, a limited scalability issue was identified as a potential challenge needing further investigation.

Another study by Kounelis et al. (2017) presented an architecture named Helios based on smart contracts. The system is designed for P2P solar power production and distribution. The focus of this application is to enable a more open and end-user centric micro-energy transaction market. An in-house testbench was created to test and validate the performance of the proposed architecture. The result showed a certain level of flexibility, but the performance in a real-world scenario still requires further testing. There is also a potential challenge regarding energy loss during transfers that also needs to be addressed by future research.

Payment systems in the P2P energy trading systems have also been investigated in recent literature. Lundqvist, de Blanche and Andersson (2017) created a single-fee micropayment system in which multiple small payments merge into one big payment to reduce the transaction cost. The report included the test result from a proof-ofconcept prototype implementation created by the researchers. According to the authors, the implementation proved that "trustless, autonomous, and ubiquitous thing to thing micropayments is no longer a future technology" (Lundqvist, de Blanche, \& Andersson, 2017 , p.6). Although this system is feasible, many obstacles still exist. For example, the prototype system was too large to be practical. The bitcoin component has limited transaction capacity, and there is a relatively high transaction fee within the bitcoin network.

A complete P2P energy trading system was designed by Cheng, Zeng, and Huang (2017). Their report presented both a decentralized P2P energy trading system, and a pricing mechanism as a tool to balance the supply and demand in the network. While their research presented a more integrated system, their report consisted only of the design and had no actual test results or future development work suggested.

Munsing, Mather, and Moura (2017) presented their design of architecture focusing on the monitoring of the payments and operations. The concept of decentralization was introduced as a solution to the trust problem in the microgrids. The design was tested on a simulated test network to evaluate the performance. However, security, transparency and robustness were presumed based on previous work, instead of actually being tested; the authors suggested further testing as a future direction.

Transaction Management Platform (TMP) is a key component of P2P energy trading systems, whose main function is to balance the supply and demand in the market. One TMP that has been discussed by various researchers is the Privacy-preserving 
Energy Transactions (PETra) (Kvaternik et al., 2017; Laszka, Dubey, Walker, \& Schmidt, 2017; Bergquist, Laszka, Sturm, \& Dubey, 2017). PETra was introduced by Laszka, Dubey, Walker and Schmidt (2017) as a solution for transactive microgrids. PETra is built on public ledgers to provide anonymity for transactions and security. Later in the same year, Kvaternik et al. (2017) further investigated into PETra and extended the existing work by analyzing the requirements and designing both architectural and protocol specifications of the blockchain-based PETra system. They evaluated their design through a case study using data obtained from Siemens. During the test, the authors identified challenges in performance, reliability and privacy, where additional off-chain support is necessary to make the implementation effective.

Additional work was done by focusing on the anonymity of the communications and transactions of PETra (Bergquist, Laszka, Sturm, \& Dubey, 2017). The outcome of the research was a design that anonymizes the complete transaction chain, providing top level of anonymity to the system. However, as this report did not mention any test results, the effectiveness of the design needs further evaluation.

\section{Grid Enhancement}

In the existing power grids, blockchain also is found helpful in increasing the security of the grids and the transparency of smart meters (Shen \& Pena-Mora, 2018). Data security is a very critical part of smart grids, as it can severely impact both energy companies and users in many ways. Faulty information stored in the system can cause the users to suffer from extra charges and can also mislead the energy companies to decisions that harm the whole system (Xie et al., 2019). Blockchain is known for its high-level data transparency, integrity and reliability, which help meet the requirements of a smart energy system effectively.

A group of researchers introduced a system aiming at enhancing the proportionalfairness control in microgrids. This is an enhancement to the microgrid and helps manage distributed energy resources (DERs) in the microgrid (Danzi, Angjelichinoski, Stefanovic, \& Popovski, 2017). The evaluation of the system was completed through a simulation, and the result showed that the blockchain prototype has the potential to enforce the security and trust of the control units. On the other hand, the researchers recommended further investigation of more complex scenarios so that the design can be improved. The authors also identified costs that need to be considered, especially mining and communication costs.

The security issue has been one of the critical concerns in smart grids. Gao et al. (2018) introduced a tamper-proof system that utilizes a blockchain to increase the safety of consumer data stored on the grid. While the authors compared their design with some existing approaches, their work remained untested and required further tests for 
performance evaluation.

Another design regarding the security sector was presented by Liang, Weller, Luo, Zhao, and Dong (2019), which focuses on increasing the resistance of the power systems against cyber-attacks. This is achieved by creating a distributed network using meters as nodes and meter measurements as blocks in the blockchain. The authors tested their model by running simulation experiments using the IEEE-118 benchmark system. The result supported the conclusion that blockchain can be applied to enhance data security in modern energy systems. However, the authors also indicated that a blockchain is resource intensive and improvements in cost and speed are essential for the system to become more reliable and competent.

Dong, Luo, and Liang (2018) investigated the enhancement of a smart grid from a slightly different perspective. They provided a conceptual model of the complete infrastructure of a smart grid, including both cyber and physical infrastructures. On the cyber side of the model, IoT, blockchain and cloud computing were combined to create a computing environment that can fulfil different requirements for future energy systems. The challenges identified by the authors included redundancy, scalability, software security, coordination, and the integration of blockchain and physical infrastructures. By the time of completion of their report, the researchers were still working on an implementation of the application, and the practical reliability was yet to be confirmed. Regardless of industry, the Cloud and Blockchain are intertwined and blockchain applications will be typically implemented and tested on cloud-based platforms (Purdon \& Erturk, 2017).

\section{Electric Vehicle (EV) Support}

Another area of interest in applying blockchain in the energy domain is in the electric vehicle industry (Shen \& Pena-Mora, 2018). Huang, Xu, Wang, and Liu (2018) argue that security problems can severely impact the use of electric vehicles. They designed a security model called LNSC (lightning network and smart contract) to enhance the security of the transaction between the EVs and the charging piles utilizing smart contract and blockchain technology. The researchers completed a set of experiments using real infrastructures for performance validation. The experiment results proved that the LNSC system is effective in enhancing the security of EV charging systems.

Another article investigated blockchain technologies for autonomous electric vehicles, and proposed architecture for EV charging (Pedrosa \& Pau, 2018). Based on their analysis of a refueling scenario, the design showed a right level of security against various types of attacks, while minimizing delay and latency. The authors used a scenario to test the performance of the architecture and found that their system meets 
most of the requirements for a smart mobility system. However, a potential risk is that, if the user set up is not done correctly, this may cause operational vulnerabilities. Also investigating the charging scheme for EVs, Liu, Chai, Zhang, Lau and Chen (2018) proposed a blockchain-based EV participation scheme called AdBEV. The primary purpose of this scheme is to reduce the fluctuation level in energy trading platforms while maintaining reliable security and anonymity level. Simulation experiments were done to evaluate system performance. The results proved the reliability of the system and showed that the algorithm successfully increased trading efficiency.

Apart from applications related to charging, another topic of interest is the billing systems for EVs. A billing system based on a lightweight blockchain was introduced by Kim, Kang, and Hong (2017) to free the system from the traditional trust-based model and provide more reliable transaction services. A shortcoming of this research was that although the authors did a projected performance analysis, the system still required practical tests to prove its effectiveness.

\section{METHODOLOGY}

\section{Research Question}

The methodology section explains the research methods, the literature search criteria, and the instruments utilized in this study. In this research, the main methodology involved is a qualitative literature review. As mentioned in the introduction, the research question of this report is:

What are the impacts of the application of blockchain technology in smart energy?

\section{Search Criteria}

The articles used in this study were identified from two other literature reviews discussing blockchain and smart environments in general. The first is the report written by Shen and Pena-Mora (2018), and the second is the report by Xie et al. (2019). The present study has formulated its unique focus and coverage, which is a recommended literature review practice (Cooper, 1988). Articles related to smart energy were selected, based on the inclusion and exclusion criteria below.

\section{Inclusion Criteria}

* Published is in English

* Published between 2016-2019

* Peer reviewed

* Paper contains designed applications and/or prototype evaluation 


\section{Exclusion Criteria}

* Papers that are focusing on concepts only

* Papers without blockchain applications in the smart energy sector

\section{Selected Articles}

The main keywords used for the search and selection of the articles were blockchain and energy. These keywords were found in one or more of the following places: article title, article abstract, and article keywords. The current study analyzed 16 scholarly articles that included Smart Energy application proposals and designs using Blockchain Technology. In a literature review, it is helpful to present the list the articles to the readers in a table with bibliometric information as in, for example, Yang, Wang, and Chen (2017). Therefore, Table 1 has been created for this purpose, sorted by the number of citations in Google Scholar as of 31 October 2019.

Table 1. Selected Articles on Smart Energy Applications Using Blockchain

\begin{tabular}{|c|c|c|c|}
\hline Authors & Year & Title & Citations \\
\hline Aitzhan and Svetinovic & 2018 & $\begin{array}{l}\text { Security and privacy in } \\
\text { decentralized energy trading } \\
\text { through multi-signatures, } \\
\text { blockchain, and anonymous } \\
\text { messaging streams }\end{array}$ & 297 \\
\hline Munsing, Mather, and Moura & 2017 & $\begin{array}{l}\text { Blockchains for decentralized } \\
\text { optimization of energy } \\
\text { resources in microgrid networks }\end{array}$ & 109 \\
\hline $\begin{array}{c}\text { Liang, Weller, Luo, Zhao, and } \\
\text { Dong }\end{array}$ & 2019 & $\begin{array}{l}\text { Distributed blockchain-based } \\
\text { data protection framework for } \\
\text { modern power systems against } \\
\text { cyber attacks }\end{array}$ & 54 \\
\hline Huang, Xu, Wang, and Liu & 2018 & $\begin{array}{l}\text { LNSC: A security model for } \\
\text { electric vehicle and charging } \\
\text { pile management based on the } \\
\text { blockchain ecosystem }\end{array}$ & 43 \\
\hline $\begin{array}{c}\text { Laszka, Dubey, Walker, and } \\
\text { Schmidt }\end{array}$ & 2017 & $\begin{array}{l}\text { Providing privacy, safety, and } \\
\text { security in IoT-based } \\
\text { transactive energy systems } \\
\text { using distributed ledgers }\end{array}$ & 42 \\
\hline Lundqvist, de Blanche, and & 2017 & Thing-to-thing electricity & 31 \\
\hline
\end{tabular}




\begin{tabular}{|c|c|c|c|}
\hline Authors & Year & Title & Citations \\
\hline Andersson & & $\begin{array}{l}\text { micropayments using } \\
\text { blockchain technology }\end{array}$ & \\
\hline $\begin{array}{c}\text { Gao, Asamoah, Sifah, Smahi, } \\
\text { and Xia }\end{array}$ & 2018 & $\begin{array}{l}\text { GridMonitoring: secured } \\
\text { sovereign blockchain-based } \\
\text { monitoring on smart grid }\end{array}$ & 31 \\
\hline $\begin{array}{l}\text { Danzi, Angjelichinoski, } \\
\text { Stefanovic, and Popovski }\end{array}$ & 2017 & $\begin{array}{l}\text { Distributed proportional- } \\
\text { fairness control in microgrids } \\
\text { via blockchain smart contracts }\end{array}$ & 25 \\
\hline $\begin{array}{c}\text { Kounelis, Steri, Giuliani, } \\
\text { Geneiatakis, Neisse, and Nai- } \\
\text { Fovino }\end{array}$ & 2017 & $\begin{array}{l}\text { Fostering consumers' energy } \\
\text { market through smart contracts }\end{array}$ & 24 \\
\hline $\begin{array}{c}\text { Liu, Chai, Zhang, Lau, and } \\
\text { Chen }\end{array}$ & 2018 & $\begin{array}{l}\text { Adaptive blockchain-based } \\
\text { electric vehicle participation } \\
\text { scheme in smart grid platform }\end{array}$ & 19 \\
\hline Dong, Luo, and Liang & 2018 & $\begin{array}{l}\text { Blockchain: a secure, } \\
\text { decentralized, trusted cyber } \\
\text { infrastructure solution for future } \\
\text { energy systems }\end{array}$ & 17 \\
\hline Kim, Kang, and Hong & 2017 & $\begin{array}{l}\text { Mobile charger billing system } \\
\text { using lightweight blockchain }\end{array}$ & 15 \\
\hline Cheng, Zeng, and Huang & 2017 & $\begin{array}{l}\text { Research on the application } \\
\text { model of blockchain technology } \\
\text { in the distributed electricity } \\
\text { market }\end{array}$ & 13 \\
\hline $\begin{array}{c}\text { Bergquist, Laszka, Sturm, and } \\
\text { Dubey }\end{array}$ & 2017 & $\begin{array}{l}\text { On the design of } \\
\text { communication and transaction } \\
\text { anonymity in blockchain-based } \\
\text { transactive microgrids }\end{array}$ & 13 \\
\hline Pedrosa and Pau & 2018 & $\begin{array}{l}\text { ChargeltUp: On blockchain- } \\
\text { based technologies for } \\
\text { autonomous vehicles }\end{array}$ & 9 \\
\hline $\begin{array}{c}\text { Kvaternik, Laszka, Walker, } \\
\text { Schmidt, Sturm, Lehofer and } \\
\text { Dubey }\end{array}$ & 2017 & $\begin{array}{l}\text { Privacy-preserving platform for } \\
\text { transactive energy systems }\end{array}$ & 7 \\
\hline
\end{tabular}

\section{Data Extraction and Storage}

As presented earlier, the research question of this study is to find the impacts of the 
application of blockchain in the smart energy domain. Both positive and negative factors need to be taken into consideration. For this purpose, the design of the data collection form divided "impact" into benefits versus risks and challenges for further analysis. To summarize information from the literature, the following data extraction table was initially used.

Table 2. Data Extraction Form

\begin{tabular}{|c|l|l|l|l|}
\hline Title & Application & Benefits & Risks \& Challenges & Design Type \\
\hline
\end{tabular}

The Application column contains a description for the application mentioned in the literature, the Benefits and Risks \& Challenges columns show the advantages and disadvantages mentioned in the literature respectively, and the Design Type column categorizes the type of technology application.

After the initial data extraction, the second round of data extraction was performed, where impact factors (benefits and challenges) were listed in a more organized way for further analysis. Two new tables were used for this purpose. The data extraction form corresponding to all of the tables were kept in MS Excel.

Table 3. Impact - Benefit

\begin{tabular}{|c|c|}
\hline Benefits & Source \\
\hline
\end{tabular}

Table 4. Impact - Risk \& Challenge Risks \& Challenges Source

This literature review has led to the findings in the following section.

\section{FINDINGS}

In the literature, many impact factors have been identified by the researchers. Some of the factors may have a positive outcome, while others may bring risks and challenges to the projects. A total of 16 articles were analyzed. Each included the proposal of an application, model, or framework. Based on the focus of the research studies and the types of these implementations, the impact factors derived from each report may seem different initially, but be similar in essence.

\section{Beneficial Factors}

The beneficial factors in the literature are categorized into seven types, including: increased security of the smart grid, improved data privacy, data and activity 
transparency and immutability, removal of third-party control and trust, cost reduction, ubiquitous solution, and greater data accessibility.

Increased security of the smart grid. The decentralized nature of blockchain technology can highly increase the security of the smart grid system, as the entire network will monitor the transactions (Aitzhan \& Svetinovic, 2018; Cheng, Zeng, \& Huang, 2017; Dong, Luo, \& Liang, 2018; Gao et al., 2018; Huang, Xu, Wang, \& Liu, 2018; Kvaternik et.al, 2017; Laszka, Dubey, Walker, \& Schmidt, 2017; Liang, Weller, Luo, Zhao, \& Dong, 2019; Liu, Chai, Zhang, Lau, \& Chen, 2018; Munsing, Mather, \& Moura, 2017; Pedrosa \& Pau, 2018).

Improved data privacy. In most smart grid cases, a public blockchain is used, in which the identity of each unit in the network remains anonymous to others. The blockchain only stores information of transactions, thus data privacy and user identity can be protected (Aitzhan \& Svetinovic, 2018; Bergquist, Laszka, Sturm, \& Dubey, 2017; Kvaternik et al., 2017; Laszka, Dubey, Walker, \& Schmidt, 2017; Lundqvist, de Blanche, \& Andersson, 2017).

Data and activity transparency and immutability. The fact that all transactions in the blockchain are broadcast to all nodes in the network ensures the transparency of transactions and activities. For the same reason, it is almost impossible to hide any individual activity from the network (Cheng, Zeng, \& Huang, 2017; Dong, Luo, \& Liang, 2018; Gao et al., 2018; Kim, Kang, \& Hong, 2017; Kounelis et al., 2017; Munsing, Mather, \& Moura, 2017).

Removal of third-party control and trust. One of the most important features of blockchain is being a decentralized system. Removing the third-party control of transactions makes the risk of third-party failure irrelevant, thereby increasing the system's reliability and stability (Cheng, Zeng, \& Huang, 2017; Danzi, Angjelichinoski, Stefanovic, \& Popovski, 2017; Kim, Kang, \& Hong, 2017; Kounelis et al., 2017; Lundqvist, de Blanche, \& Andersson, 2017).

Cost reduction. Blockchain technology may reduce transaction costs, for example, reducing the transaction fee, and reducing the number of failed transactions (Kim, Kang, \& Hong, 2017; Kounelis et al., 2017; Liu, Chai, Zhang, Lau, \& Chen, 2018).

Ubiquitous solution. Some researchers believe that a blockchain-based smart grid system can be a ubiquitous solution in order to address many different smart energy problems (Dong, Luo, \& Liang, 2018; Lundqvist, de Blanche, \& Andersson, 2017). 
Greater data accessibility. The blockchain structure determines that, once a block is added to the chain, it can no longer be edited or deleted. Data is always accessible after it is logged into the chain (Cheng, Zeng, \& Huang, 2017; Kim, Kang, \& Hong, 2017; Kvaternik et al., 2017).

Negative factors related to blockchain technology also need to be considered. The following section presents the risks and challenges identified in the selected literature.

\section{Risk and Challenge Factors}

The significant risks and challenges in the literature for blockchain-based smart energy applications include limited scalability and speed, off-chain support requirement, high establishment and maintenance cost, high transaction cost, and requiring further practical tests.

Limited scalability and speed. The limitation on scalability and speed is a widely recognized issue and challenge with current blockchain technology in general. For example, Bitcoin has a minimal rate of around seven transactions per second, due to the amount of work required for PoW. In comparison, the Visa transaction system can support an average of 2000 transactions per second (Aitzhan \& Svetinovic, 2018; Danzi, Angjelichinoski, Stefanovic, \& Popovski, 2017; Dong, Luo, \& Liang, 2018; Kvaternik et al., 2017; Liang, Weller, Luo, Zhao, \& Dong, 2019; Lundqvist, de Blanche, \& Andersson, 2017; Munsing, Mather, \& Moura, 2017).

Off-chain support requirement. A smart energy system cannot function without the proper set up of physical infrastructures. While blockchain can help improve the quality of the digital record keeping, it does not improve the original data input. This brings up the requirement of a well-designed and operated off-chain system, to ensure that the system's raw data input is correct and reliable (Aitzhan \& Svetinovic, 2018; Dong, Luo, \& Liang, 2018; Kvaternik et al., 2017; Liu, Chai, Zhang, Lau, \& Chen, 2018; Pedrosa $\&$ Pau, 2018).

High establishment and maintenance cost. Another downside of blockchain identified by the literature is the relatively high cost in initial setup and maintenance. Since blockchain is a decentralized system, and slightly different from most traditional systems, the cost of implementation may be a problem in many cases (Danzi, Angjelichinoski, Stefanovic, \& Popovski, 2017; Dong, Luo, \& Liang, 2018; Liang, Weller, Luo, Zhao, \& Dong, 2019; Pedrosa \& Pau, 2018). 
High transaction cost. While some researchers believe that applying blockchain for a smart energy system can eventually lead to a lower transaction cost, others argue that it would result in a higher transaction cost (Danzi, Angjelichinoski, Stefanovic, \& Popovski, 2017; Kounelis et al., 2017; Lundqvist, de Blanche, \& Andersson, 2017).

Requiring further practical tests. Lack of past practical tests is a challenge many researchers faced in their studies. Although many of the proposed systems functioned properly under test benches and simulations, a large-scale test in a real-world context will provide more reliable results and may reveal other issues (Gao et al., 2018; Kounelis et al., 2017; Danzi, Angjelichinoski, Stefanovic, \& Popovski, 2017; Lundqvist, de Blanche, \& Andersson, 2017).

In this section, the impact of blockchain technology in the smart energy domain identified in the literature was analyzed. The research suggested seven positive factors: increased security of the smart grid, improved data privacy, data and activity transparency and immutability, removal of third-party trust, cost reduction, ubiquitous solution, and data accessibility. On the other hand, five significant risks and challenges were recognized by the researchers: limited scalability and speed, off-chain support requirement, high establishment and maintenance cost, high transaction cost, and need for practical tests. The following table provides a summary of the key findings.

Table 5. Summary of the Findings

\begin{tabular}{|l|c|c|}
\hline Factor & $\begin{array}{l}\text { Type of } \\
\text { Impact }\end{array}$ & $\begin{array}{c}\text { Number of Related } \\
\text { Articles (out of 16) }\end{array}$ \\
\hline Increased security of the smart grid & Benefit & 11 \\
\hline Improved data privacy & Benefit & 5 \\
\hline Data and activity transparency and immutability & Benefit & 6 \\
\hline Removal of third-party trust & Benefit & 5 \\
\hline Cost reduction & Benefit & 3 \\
\hline Ubiquitous solution & Benefit & 2 \\
\hline Data accessibility & Benefit & 3 \\
\hline Limited scalability and speed & Challenge & 7 \\
\hline Off-chain support requirement & Challenge & 5 \\
\hline High establishment and maintenance cost & Challenge & 4 \\
\hline High transaction cost & Challenge & 3 \\
\hline Practical test & Challenge & 3 \\
\hline
\end{tabular}


The next section will discuss these findings, further exploring the relationships revealed by the results shown in this section.

\section{DISCUSSION}

During the review, the most commonly identified beneficial factor is the increase in the security of the smart grid, with 11 articles mentioning it in different ways (Aitzhan \& Svetinovic, 2018; Cheng, Zeng \& Huang, 2017; Dong, Luo, \& Liang, 2018; Gao et al., 2018; Huang, Xu, Wang, \& Liu, 2018; Kvaternik et al., 2017; Laszka, Dubey, Walker, \& Schmidt, 2017; Liang, Weller, Luo, Zhao, \& Dong, 2019; Liu, Chai, Zhang, Lau, \& Chen, 2018; Munsing, Mather, \& Moura, 2017; Pedrosa \& Pau, 2018). This result is understandable, as security is a common requirement for the majority of the systems. Another popular feature of Blockchain Technology (with a total of 11 studies mentioning it) is improving data management. These relevant beneficial factors are improved data privacy and increased data transparency/immutability, with five for the first part (Aitzhan \& Svetinovic, 2018; Bergquist, Laszka, Sturm, \& Dubey, 2017; Kvaternik et al., 2017; Laszka, Dubey, Walker, \& Schmidt, 2017; Lundqvist, de Blanche, \& Andersson, 2017) and six for the latter (Cheng, Zeng, \& Huang, 2017; Dong, Luo, \& Liang, 2018; Gao et al., 2018; Kim, Kang, \& Hong, 2017; Kounelis et al., 2017; Munsing, Mather, \& Moura, 2017).

Most applications in the literature involved processing transactions, and most of the users are from the general public. Therefore, data privacy of the transactions and especially protecting personal identity become critical. Blockchain can provide a satisfying level of data privacy through its pseudo-anonymous nature. Data transparency on the other hand, can significantly enhance the monitoring of the systems. Three other research studies mentioned the high accessibility provided by blockchain as a benefit. Their applications demand strict monitoring of the system, where data accessibility is a critical concern. Another one of blockchain's unique feature is the removal of third-party management. This is recognized as an advantage by five articles (Cheng, Zeng, \& Huang, 2017; Danzi, Angjelichinoski, Stefanovic, \& Popovski, 2017; Kim, Kang, \& Hong, 2017; Kounelis et al., 2017; Lundqvist, de Blanche, \& Andersson, 2017). Two of the research studies recommended that blockchain-based applications can be used as a ubiquitous solution for future energy system (Dong, Luo, \& Liang, 2018; Lundqvist, de Blanche, \& Andersson, 2017).

On the other hand, from the risk and challenges point of view, a highly recognized challenge is the limitation of blockchain technology in terms of scalability and speed. Seven articles found this limitation to be an obstacle for the practical application of blockchain in real-world scenarios and recommend that future improvement of this technology is to be expected (Aitzhan \& Svetinovic, 2018; Danzi, Angjelichinoski, 
Stefanovic, \& Popovski, 2017; Dong, Luo, \& Liang, 2018; Kvaternik et.al, 2017; Liang, Weller, Luo, Zhao, \& Dong, 2019; Lundqvist, de Blanche, \& Andersson, 2017; Munsing, Mather, \& Moura, 2017). Whereas blockchain is only a digital tool, some applications involve both physical and digital infrastructures. These examples are indicative that the proper setup of corresponding off-chain support is another critical challenge. Four groups of researchers suggest that the risk of using a low-quality off-chain physical infrastructure needs to be considered carefully, as blockchain is not necessarily supposed to decide whether the information received is correct or not.

Figure 1 briefly summarizes and illustrates the findings of this paper. Lower transaction costs were suggested by some of the papers as one of the seven beneficial factors; while other papers identified higher transaction costs as one of the five risks. Therefore, the impact on transaction costs is shown in the center as "unclear" below.
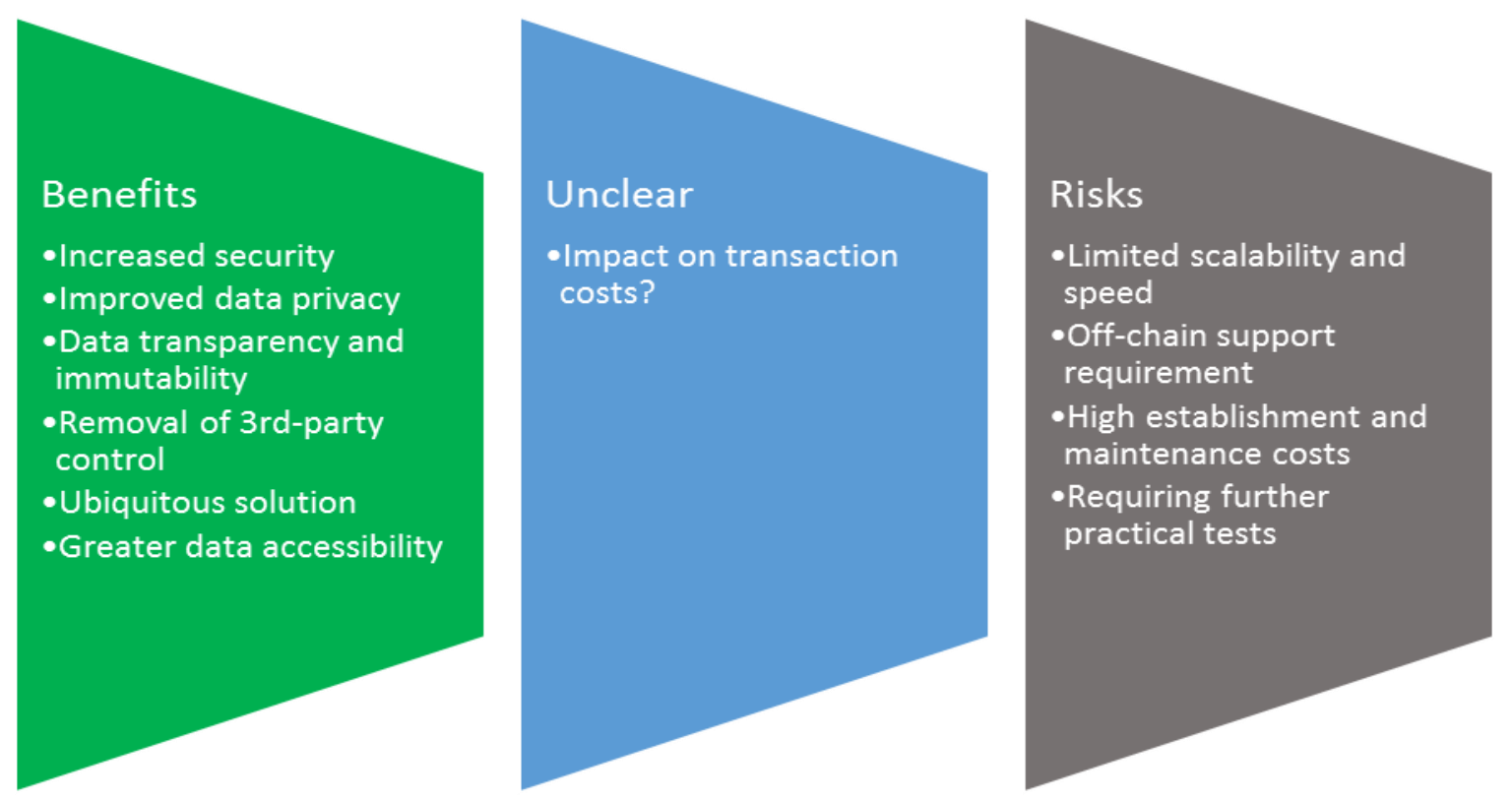

Figure 1. Blockchain Technology in Smart Energy

The impact of blockchain on cost is a complex topic. There is not a general consensus whether blockchain can reduce the cost or not. The circumstances may vary and the role of the blockchain may be different, depending on the system. In the P2P energy trading industry, Kounelis et al. (2017) believed that blockchain is double-edged in terms of costs. The removal of third-party involvement may reduce the cost by a large amount, while it may also increase the energy use during transactions, if the system logic and transaction schemes are not efficiently designed. In electric vehicle charging systems on the other hand, two studies recommended blockchain as a solution to reduce the cost of the systems. Another concern in term of blockchain's impact on cost is the potentially high implementation and maintenance costs. Four articles identified that for 
a blockchain-based system, the establishment cost can be significant, both in terms of introducing new infrastructures and of increasing user involvement in the new system. How blockchain may impact the cost of the system is dependent on the case itself. With proper design and setup, blockchain can be beneficial in the long term. But with poor design and lack of communication between the involved parties, blindly applying blockchain could eventually cause an increase in costs.

From the design type point of view, it is expected that the increase in data security is an agreed benefit for all types of applications. The same was also noticed for data transparency and immutability increase, which appeared to be an attractive feature for all types of applications. However, unexpectedly, improvement in data privacy was reported as a benefit by only one $\mathrm{P} 2 \mathrm{P}$ energy trading application. This might be a result of the scale of the database, or it might happen because other interface applications do not enforce such a high level of privacy. Removal of third-party control and trust is recognized among $\mathrm{P} 2 \mathrm{P}$ energy trading and grid enhancement applications, despite no mention for electric vehicle related applications. A possible reason could be that the particular researchers did not yet consider business factors.

From the perspective of challenges, off-chain support requirements and high establishment and maintenance costs were both suggested by all three types of applications. Since blockchain can only protect the records on the chain, it is necessary for the physical infrastructures to guarantee the correctness of the input (Shen \& PenaMora, 2018). Limitations of blockchain technology in terms of speed and scalability is a challenge identified for $\mathrm{P} 2 \mathrm{P}$ energy trading and grid enhancement applications. Electric vehicle related applications in this study did not report this issue, possibly because the scale of the tests was not large and there is not a large number of electric vehicles yet. For example, in New Zealand, only 14,095 out of 5 million registered vehicles are electric vehicles ("National Vehicle Fleet status | NZ Transport Agency", 2019; "Monthly electric and hybrid light vehicle tables | Ministry of Transport", 2019).

\section{CONCLUSION}

This report provided an analysis of blockchain technology applications in the smart energy domain. The discussion began with an introduction to the essential concepts and background knowledge of blockchain technology and smart energy. Then some applications of blockchain-based smart energy systems were introduced with more details, including the contents of the applications and the strategy used for evaluation. This study analyzed both the positive and negative impact of blockchain technology in smart energy. The design types of the applications were also recognized in conjunction with the positive and negative impact of blockchain.

Future systematic literature reviews can look at similar blockchain benefits and 
challenges with new articles and possibly using a broader sample. The unclear impact regarding transaction costs was an interesting finding of this study. Consequently, there is an opportunity for future quantitative studies to measure and analyze the impact of this technology on economic feasibility and transaction costs.

In summary, the application of blockchain in smart energy is a complex topic and there are still many challenges. Another future research direction is to compare the performance of existing operational blockchain-based smart energy systems against the theories and recommendations in the literature. This can help create a stronger link between the smart energy industry and the research.

\section{REFERENCES}

Aitzhan, N., \& Svetinovic, D. (2018). Security and privacy in decentralized energy trading through multi-signatures, blockchain and anonymous messaging streams. IEEE Transactions on Dependable and Secure Computing, 15(5), 840-852. https://doi.org/10.1109/tdsc.2016.2616861

Bergquist, J., Laszka, A., Sturm, M., \& Dubey, A. (2017). On the design of communication and transaction anonymity in blockchain-based transactive microgrids. Proceedings of the 1St Workshop on Scalable and Resilient Infrastructures for Distributed Ledgers - SERIAL '17.

https://doi.org/10.1145/3152824.3152827

Cheng, S., Zeng, B., \& Huang, Y. (2017). Research on application model of blockchain technology in distributed electricity market. IOP Conference Series: Earth and Environmental Science, 93, 012065.

https://doi.org/10.1088/1755-1315/93/1/012065

Cooper, H. (1988). Organizing knowledge syntheses: A taxonomy of literature reviews. Knowledge in Society, 1(1), 104-126. https://doi.org/10.1007/bf03177550

Crosby, M., Nachiappan, M., Pattanayak, P., Verma, S., \& Kalyanaraman, V. (2016). BlockChain technology: Beyond bitcoin. Applied Innovation Review, 2, 8-19.

Danzi, P., Angjelichinoski, M., Stefanovic, C., \& Popovski, P. (2017). Distributed proportional-fairness control in microgrids via blockchain smart contracts. 2017 IEEE International Conference on Smart Grid Communications (Smartgridcomm). https://doi.org/10.1109/smartgridcomm.2017.8340713

Dong, Z., Luo, F., \& Liang, G. (2018). Blockchain: A secure, decentralized, trusted cyber infrastructure solution for future energy systems. Journal of Modern Power Systems and Clean Energy, 6(5), 958-967. https://doi.org/10.1007/s40565-0180418-0

El Rahi, G., Saad, W., Glass, A., Mandayam, N., \& Poor, H. (2016). Prospect theory for prosumer-centric energy trading in the smart grid. 2016 IEEE Power \& Energy 
Society Innovative Smart Grid Technologies Conference (ISGT).

https://doi.org/10.1109/isgt.2016.7781198

Ethereum Foundation. (2019). What is Ethereum? Retrieved from https://www.ethereum.org/beginners/

Gao, J., Asamoah, K., Sifah, E., Smahi, A., Xia, Q., \& Xia, H. et al. (2018). GridMonitoring: Secured Sovereign Blockchain Based Monitoring on Smart Grid. IEEE Access, 6, 9917-9925. https://doi.org/10.1109/access.2018.2806303

Huang, X., Xu, C., Wang, P., \& Liu, H. (2018). LNSC: A Security Model for Electric Vehicle and Charging Pile Management Based on Blockchain Ecosystem. IEEE Access, 6, 13565-13574. https://doi.org/10.1109/access.2018.2812176

Linux Foundation. (2019). Hyperledger Fabric. Retrieved from https://www.hyperledger.org/projects/fabric

Jenkins, N., Long, C., \& Wu, J. (2015). An overview of the smart grid in Great Britain. Engineering, 1(4), 413-421. https://doi.org/10.15302/J-ENG-2015112

Kim, N., Kang, S., \& Hong, C. (2017). Mobile charger billing system using lightweight Blockchain. 2017 19Th Asia-Pacific Network Operations and Management Symposium (APNOMS). https://doi.org/10.1109/apnoms.2017.8094151

Kounelis, I., Steri, G., Giuliani, R., Geneiatakis, D., Neisse, R., \& Nai-Fovino, I. (2017). Fostering consumers' energy market through smart contracts. 2017 International Conference in Energy and Sustainability in Small Developing Economies (ES2DE). https://doi.org/10.1109/es2de.2017.8015343

Kvaternik, K., Laszka, A., Walker, M., Schmidt, D., Sturm, M., Lehofer, M., and Dubey, A. (2017). Privacy-Preserving Platform for Transactive Energy Systems.

Laszka, A., Dubey, A., Walker, M., \& Schmidt, D. (2017). Providing privacy, safety, and security in IoT-based transactive energy systems using distributed ledgers. Proceedings of The Seventh International Conference 0n The Internet of Things Iot '17. https://doi.org/10.1145/3131542.3131562

Liang, G., Weller, S., Luo, F., Zhao, J., \& Dong, Z. (2019). Distributed blockchainbased data protection framework for modern power systems against cyber attacks. IEEE Transactions on Smart Grid, 10(3), 3162-3173.

https://doi.org/10.1109/tsg.2018.2819663

Liu, C., Chai, K., Zhang, X., Lau, E., \& Chen, Y. (2018). Adaptive blockchain-based electric vehicle participation scheme in smart grid platform. IEEE Access, 6, 25657-25665. https://doi.org/10.1109/access.2018.2835309

Lundqvist, T., de Blanche, A., \& Andersson, H. (2017). Thing-to-thing electricity micro payments using blockchain technology. 2017 Global Internet of Things Summit (Giots). https://doi.org/10.1109/giots.2017.8016254

Ministry of Transport. (2019). Monthly electric and hybrid light vehicle tables. 


\section{Retrieved from}

https://transport.govt.nz/mot-resources/vehicle-fleet-statistics/monthly-electricand-hybrid-light-vehicle-registrations-2/

Moretti, M., Djomo, S., Azadi, H., May, K., De Vos, K., Van Passel, S., \& Witters, N. (2017). A systematic review of environmental and economic impacts of smart grids. Renewable and Sustainable Energy Reviews, 68, 888-898.

https://doi.org/10.1016/j.rser.2016.03.039

Munsing, E., Mather, J., \& Moura, S. (2017). Blockchains for decentralized optimization of energy resources in microgrid networks. 2017 IEEE Conference on Control Technology and Applications (CCTA).

https://doi.org/10.1109/ccta.2017.8062773

Nakamoto, S. (2008). Bitcoin: A Peer-to-Peer Electronic Cash System. Retrieved from https://bitcoin.org/en/bitcoin-paper

National Vehicle Fleet status | NZ Transport Agency. (2019). Retrieved from https://www.nzta.govt.nz/resources/new-zealand-motor-vehicle-registerstatistics/national-vehicle-fleet-status/

Pedrosa, A., \& Pau, G. (2018). ChargeltUp: On Blockchain-based technologies for Autonomous Vehicles. Proceedings of the 1St Workshop on Cryptocurrencies and Blockchains for Distributed Systems - Cryblock'18.

https://doi.org/10.1145/3211933.3211949

Purdon, I., \& Erturk, E. (2017). Perspectives of blockchain technology, its relation to the cloud and its potential role in computer science education. Engineering, Technology \& Applied Science Research, 7(6), 2340-2344.

Shen, C., \& Pena-Mora, F. (2018). Blockchain for Cities-A Systematic Literature Review. IEEE Access, 6, 76787-76819. https://doi.org/10.1109/access.2018.2880744

Strengers, Y. (2013). Smart energy technologies in everyday life. Basingstoke: Palgrave Macmillan.

Swan, M. (2015). Blockchain. Sebastopol, CA: O'Reilly Media, Inc.

Xie, J., Tang, H., Huang, T., Yu, F., Xie, R., Liu, J., \& Liu, Y. (2019). A Survey of Blockchain Technology Applied to Smart Cities: Research Issues and Challenges. IEEE Communications Surveys \& Tutorials, 1-1. https://doi.org/10.1109/comst.2019.2899617

Yang, Y. J., Wang, C. C., \& Chen, C. C. (2017). Recent Development Trend of Electronic Commerce Research: 2000 to 2016. Contemporary Management Research, 13(2). https://doi.org/10.7903/cmr.17824 
Yli-Huumo, J., Ko, D., Choi, S., Park, S., \& Smolander, K. (2016). Where is current research on blockchain technology? - A systematic review. PLOS ONE, 11(10), e0163477. https://doi.org/10.1371/journal.pone.0163477

Dr. Emre Erturk (Corresponding author) is currently a Principal Academic Staff Member at the Eastern Institute of Technology (New Zealand), School of Computing, Hawke's Bay Campus. His current research interests include Cloud Computing and Blockchain applications in business and education. He has previously published and presented more than fifty articles.

Dr. Dobrila Lopez is currently a Principal Academic Staff Member at the Eastern Institute of Technology (New Zealand), Auckland Campus. She is currently involved in a large research project to develop and increase peer mentoring among international students.

Mr. Wei Yang Yu is currently studying in the Eastern Institute of Technology's Master of Information Technology program at the Auckland Campus. He is researching Blockchain and other emerging technologies in business. 